\title{
Motivation Building Strategies of Mathematics Instruction for Undergradu- ate Students in Mechanical Engineering
}

\section{Dr. Amitabha Ghosh, Rochester Institute of Technology (COE)}

Dr. Amitabha Ghosh is a licensed Professional Engineer with a Ph.D. in general engineering composite (Major: Aerospace Engineering) from Mississippi State University. He obtained his B.Tech. and M.Tech. degrees in Aeronautical Engineering from Indian Institute of Technology, Kanpur. He is a professor of Mechanical Engineering at Rochester Institute of Technology. His primary teaching responsibilities are in the areas of fluid mechanics and aerodynamics. He is also a significant contributor in teaching of the solid mechanics courses. For the past ten years, he has been involved heavily in educational research at RIT and has also served as the coordinator of the Engineering Sciences Core Curriculum (ESCC) in Mechanical Engineering and various other curricular committees. 


\title{
Motivation Building Strategies of Mathematics Instruction for Undergraduate Students in Mechanical Engineering
}

\begin{abstract}
One of the biggest challenges in teaching upper level mathematics-oriented courses such as Fluid Dynamics or, Aerodynamics to mechanical engineering students today is the lack of focus and motivation. The advantages offered by computing tools today have simultaneously created new challenges at the undergraduate instructional level regarding understanding and absorption of theoretical modeling concepts. To compound the picture even more, lack of time and career goals have modified educational priorities in our students and pose difficulties in integrating mathematics with engineering. This paper discusses methods to overcome such barriers without compromising the mathematical rigor in learning concepts of applied mathematics. This research is supported by implanted strategies in a sequence of courses leading to and culminating in an upper level course in computational fluid dynamics.

There are many alternate outcomes posed by a flipped classroom structure adopted by many professors. Past ten years of observed data do not demonstrate deeper understanding of logical and analytical models for several reasons which are discussed in the paper. Therefore, this paper focuses on motivation building aspects to offer connectivity and slow absorption of applied mathematical tools. This research identifies the primary needs of building conceptual inventories and contrasts their differences from the theories of learning mathematics. In practice, delivering mathematical theories necessary at the upper undergraduate level in mechanical engineering is not well executed and some adjustments are necessary primarily at the lower levels. Scaffolding with conceptual inventories must be completed with enough time for sufficient reinforcement. The results realized in teaching at upper levels are derived from mathematical concepts and identifiers reinforced during core courses in mechanical engineering. The paper demonstrates such measured steps together with motivation builders as the prescription required in teaching calculus and boundary value problems to mechanical engineering students.
\end{abstract}

\section{Introduction}

On a recent examination in Dynamics given to 60 students in two sections team-taught by two different instructors, the following question was posed (Figure 1). The question tests the rigid body kinematics area covering dynamical concepts of fixed axis rotations in belt drives. The mathematically relevant part of the solution requires a simple integration with proper separation of variables using the definition of angular acceleration to obtain $\omega(\mathrm{t})$. All formulae from the textbook necessary to solve the questions were provided on a single formula sheet copied from 
the inside cover of Hibbeler [1]. Figure 1 also shows on the right the expected solution. For this discussion, only part (a) of the question is presented below.

The vacuum cleaner's armature shaft $S$ rotates with an angular acceleration of $\alpha=4 \omega \omega^{3 / 4} \mathrm{rad} / \mathrm{s}^{2}$, where $\omega$ is in rad/s. If the shaft starts from $\omega_{0}=1 \mathrm{rad} / \mathrm{s}$ at $\theta=0$, (a) find the brush's angular velocity when $t=4 \mathrm{~s}$. The radii of the shaft and the brush are $0.25 \mathrm{in}$., and $1 \mathrm{in}$. respectively, ....

Show all steps how you reached the solution. [Subscripts S, A used for shaft, roller below]

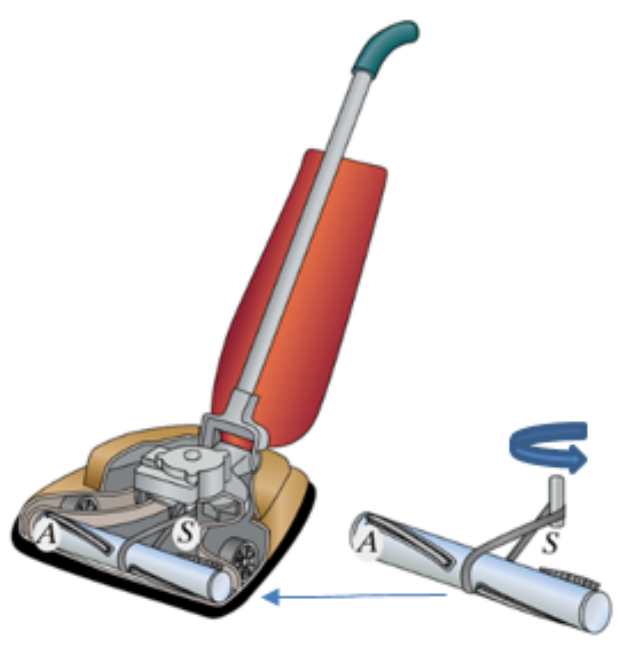

Given: $\alpha_{\mathrm{S}}=4 \omega_{\mathrm{S}}^{3 / 4}$, At $\mathrm{t}=0, \theta_{\mathrm{S}}=0, \omega_{\mathrm{S}}=1 \mathrm{rad} / \mathrm{s}$.

$r_{A}=1$ in., $r_{S}=1 / 4$ in. Find: $(a) \omega_{A}$ at $t=4 s, \ldots .$.

Since $\alpha=\frac{d \omega}{d t}=4 \omega^{3 / 4}$, for $\mathrm{S}, \int \omega_{\mathrm{S}}^{-3 / 4} \mathrm{~d} \omega_{\mathrm{S}}=4 \int \mathrm{dt}$.

Since the variables are separated already, integrate both sides to get $\omega_{\mathrm{S}}(\mathrm{t})$ first.

$\frac{\omega_{S}-\frac{3}{4}+1}{-\frac{3}{4}+1}=4 \mathrm{t}+\mathrm{C}$, where, $\mathrm{C}$ is a constant.

Evaluate $\mathrm{C}$ using the initial condition gives $\mathrm{C}=4$

$\therefore$ At $\mathrm{t}=4 \mathrm{~s}, \omega_{\mathrm{S}}=625 \mathrm{rad} / \mathrm{s}$. Use $\omega_{\mathrm{A}} \mathrm{r}_{\mathrm{A}}=\omega_{\mathrm{S}} \mathrm{r}_{\mathrm{S}} \Rightarrow$

$\omega_{\mathrm{A}}=156.25 \mathrm{rad} / \mathrm{s}$ at $\mathrm{t}=4 \mathrm{~s}$. (Answer to part (a))

Figure 1. Dynamics question (with solution) requires use of the separation of variables

Figure 2 shows samples of incorrect responses given by more than $80 \%$ of students. While $60 \%$ of students did not perform a proper separation of variables before integration (as seen from the answers of eight students), $20 \%$ of students used the (equally inapplicable) constant acceleration formula. 


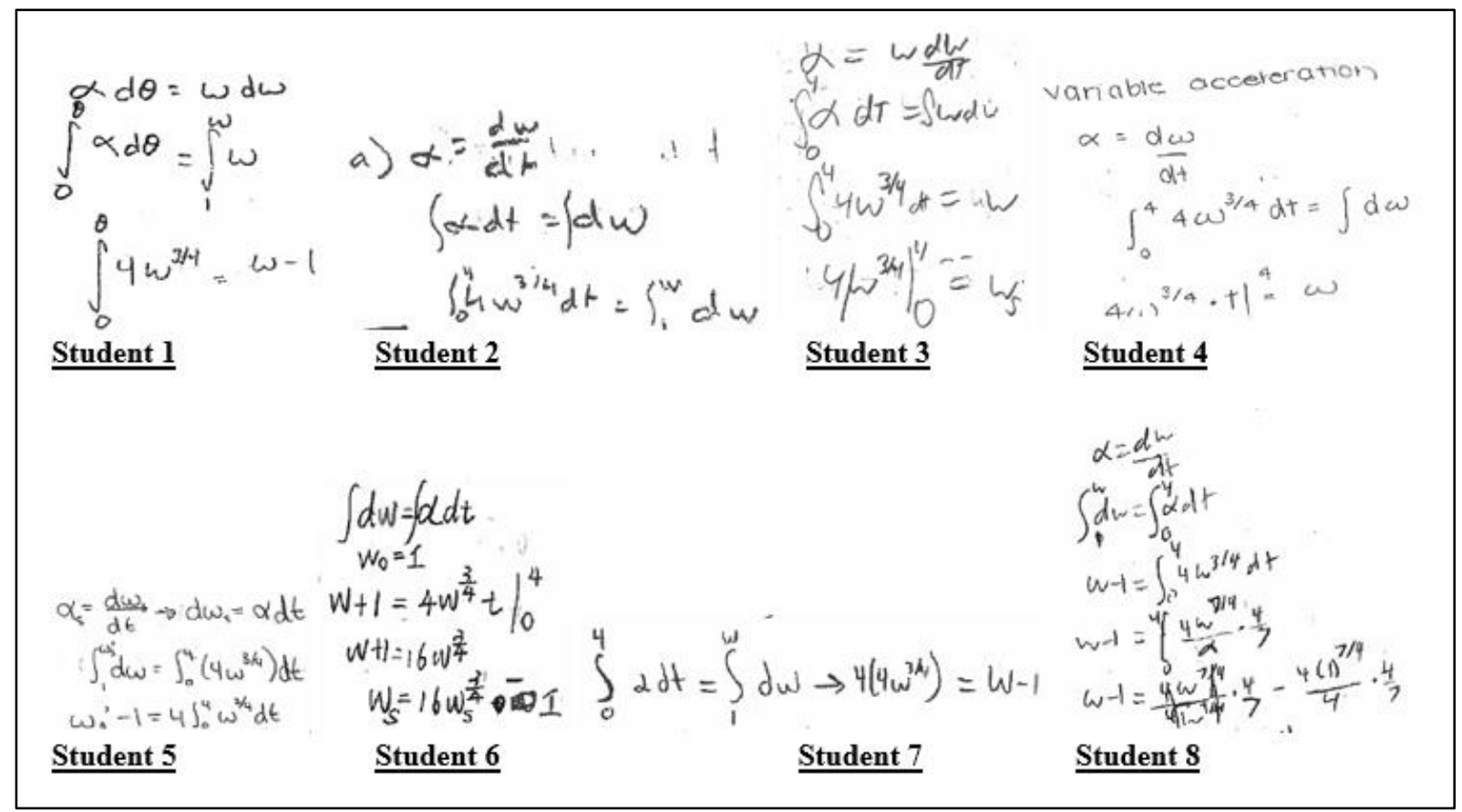

Figure 2: Typical student responses to the mathematical integration posed on Figure 1

Answers such as the ones presented above are typically received in many engineering programs today. Such failures may be blamed in part on the current advances in technology. However, in our program we collect and document identifiable data which probe deeper into the causes of failure [2], [3], [4]. Both instructors had reviewed and reinforced the relevant mathematical concepts in their respective classes because this weakness is a known area of focus in our selfassessment process [3], [5]. In this research feedback we discuss a procedure which helps retention of such mathematical concepts.

Our Mechanical Engineering (ME) curriculum tracks examination performance of students from freshman through junior levels using a carefully redesigned curriculum of engineering science core courses (ESCC) and a blended set of applied laboratories. ESCC consists of six core courses taught by teams of ME faculty with clearly set educational objectives and managed by a coordinator and trained teaching assistants. Though essay type examination questions can demonstrate positive learning outcomes, multiple choice questions are better to pinpoint areas of conceptual difficulties. After designing and adopting ESCC in 2006, faculty agreed that carefully designed multiple choice questions should form an integral part for all examinations in core classes. We frequently discuss performance data on conceptual questions and archive them with their question banks annually. The details of ESCC are given in [5], [6]. Reference [5] recently summarized the performance and recommendations in the solid mechanics sequence. Thermofluids sequence focuses more on the different approaches to mathematical modeling and aspects of competency-based learning, some of which were also described in references [4], [6].

Mathematics based flow modeling was the only recourse to learn fluid mechanics for much of human history. Till the advent of high speed computers, the only means to verify the models 
were by extensive experimentation. With the help of computational fluid dynamics (CFD) expensive experimentation has been cut to a minimum even at research levels [7, 8]. Alternate methods of optimizing productivity have been developed with the introduction of design of experiments [7]. At the education level, computer graphics and supercomputing brought the most significant impacts [9], [10]. Problems which could not be conceived as solvable before are being routinely completed with machines which may be held in the palm of a human hand. The enhanced usage and comfort however produced some new challenges at the undergraduate instructional level [4]. Today Internet, I-phones, I-pads, and social media steal much more time from our conventional undergraduate education system [11]. In this paper we focus only on the challenges in absorption and retention of mathematical concepts, whereas the engineering concept inventory will be presented elsewhere. In the past three decades educational research developed many thinking and learning theories [12] - [15], [16] - [18]. In engineering education, Self-directed competency-based learning (CBL) is quite popular today. We discuss here a blend of CBL [15] with conventional pedagogy and suggest a scaffolding process for ME students to retain mathematical concepts at the undergraduate level.

Various motivation building strategies have been practiced by our teams with different modes of delivery over the years [4], [6], [19] - [21]. These attempts achieved positive results with group incentives and cooperative learning strategies [6]. But current challenges [4] require a wider, more organized team approach and discussions in an open forum. This paper first identifies through examples the areas where mathematical rigor is necessary. Then it presents the emphasis on select topics and the advantages and drawbacks of specific pedagogy. Finally, a blended and extended approach is suggested as a hopeful remedy for better absorption of mathematical concepts. The steps proposed must start from the freshman level and reinforced through the senior level, and measured outcomes must be realizable before graduation from the BS program.

\section{Approach}

ESCC provides an approach to gather examination data as a direct evidence of learning using carefully designed conceptual questions. Together with this, we collect inputs from faculty advisors and teaching assistants to provide further proofs of identifying difficult conceptual areas from a student's viewpoint. We quote a few of these areas of mathematics which are critical from an engineering student's needs (cf. Figures 3, 4). Previously ESCC approach was focused on curricular improvements through accurate testing methods and collaborative improvement in pedagogy. Our approach discussed here is different from a standard mathematics course in differential and integral calculus presented to students of mathematics. Instead ours is an application-oriented gaged method of reinforcement with incentives. It connects mathematical concepts necessary to understand, operate and design an engineering application. For example, in the previous question from Dynamics (cf. Figure 1), the vacuum cleaner mechanism was blended on purpose with the testing of calculus, together with the point of focus (whether students know how to separate variables correctly before integration). But our experience revealed much more information about the recent study patterns of our students prompting the preparation of the current approach. 
A typical flow example may be seen on figure 3. This question was posed as a part of question 1 on the second midterm examination in Fluids II, which is an upper level elective course.

\section{Be very brief and precise in answering concept questions which has 5 parts of 10 points each.}

1. (a) A velocity profile on a wall $(\mathrm{y}=0)$ is given by $u(y)=y-\frac{y^{2}}{0.2}, v=0(\mathrm{~m} / \mathrm{s})$. Find the volumetric flowrate per unit depth in this flow, if the flow channel extends 0.2-m from the wall.

\section{Figure 3: Failure to identify correct order of mathematical operations}

$80 \%$ of the class interpreted the above velocity profile as $\mathrm{u}(\mathrm{y})=\left(\mathrm{y}-\mathrm{y}^{2}\right) / 0.2$ instead of the way the question intended. In the past five years such errors have received a new attention from ESCC faculty.

We continued with velocity profiles and asked students to evaluate shear stress on boundaries (Figure 4). We suspected such efforts would fail because most students do not connect to the velocity profiles and the information built in them. In this question students mainly struggled with the parts (c) and (d) trying to enforce the $\tau=\mu \frac{V}{h}$ formula for Couette flows, which they had learnt from the prerequisite course taught by a different instructor. The flipped class structure adopted by the other instructor apparently worked well in retaining the Couette flow shear formula. A similar inapplicability issue was observed before (cf. Figure 1). A few of the students also struggled with the units and got incorrect answers. The question demonstrates what is typically overlooked from a mathematician's viewpoint but is very important from the ME stand point. The units error emerges from the fact that the numbers 8 and 0.3 in the velocity profile have different units but when derivatives are evaluated the stress expression would emerge in $\mathrm{N} / \mathrm{m}^{2}$ (or $\mathrm{Pa}$ ) without converting the units on $\mu$.

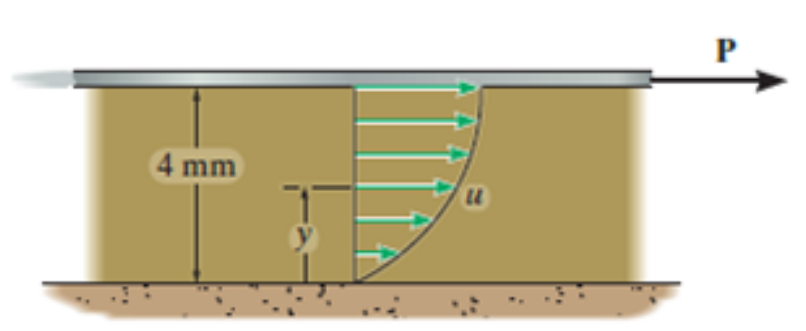

The Newtonian fluid, confined between a moving plate on top and a stationary flat surface on the bottom, develops the velocity profile: $\mathrm{u}(\mathrm{y})=\left(8 \mathrm{y}-0.3 \mathrm{y}^{2}\right) \mathrm{mm} / \mathrm{s}$, where, $\mathrm{y}$ is expressed in $\mathrm{mm}$.

(a) Sketch on the figure the origin and the coordinate axes which were used to express $u(y)$.

(b) With what speed $(\mathrm{mm} / \mathrm{s})$ is the top plate moving?

(c) Which law of viscosity would you use to find the shear stress (in $\mathrm{N} / \mathrm{m}^{2}$ ) on the top plate? Show calculation details how you'd find the shear stress on the top plate. The dynamic viscosity of the fluid $\mu=0.482 \mathrm{~N} . \mathrm{s} / \mathrm{m}^{2}$.

(d) Find the shear stress on the bottom surface from the fluid.

Figure 4: Working with velocity profiles 
After experiencing such rudimentary failures recently, we now recommend that ESCC courses should avoid a flipped structure for the following reasons. The most important flaw - it may reinforce incorrect logic and misunderstanding acquired in the self-learning process. This becomes much harder to correct later. In a group learning environment, students tend to gravitate toward choosing their own friends as teammates. If a functional group is not properly balanced with complementary skills, incorrect reasoning, inapplicable formulations and damaging shortcuts are learnt. A flipped course structure is excellent for learning mathematical skills at lower levels where information may be searched, not necessarily understood. Flipped classes are favored by many ESCC instructors today [21]. But at upper levels discussed here, a flipped class structure has to be modified considerably [22]. When upper level engineering thoughts develop, logic [6] and constraint-based scaffolding [23] must be provided by an experienced instructor. We present some examples here to elucidate.

Average ME students do not realize (while recalling the definition of slope) that working with $\mathrm{u}(\mathrm{y})$ is different from working with $\mathrm{f}(\mathrm{x})$ in the calculus class. The slope is measured here relative to the ordinate (since the dependent variable, $\mathrm{u}$ is represented as abscissa and the independent variable, $y$ is represented as ordinate). Discussions of velocity and temperature profiles in Fluids II correct and/or reinforce these concepts. Similar examples exist in later uses of need-based coordinate systems in dynamics which carry over to fluid mechanics [4]. Considerable effort must be made to stop students from memorizing mathematical techniques. Initial attempts to achieve this last feat meets some student resistance but eventually the effort develops a nice rapport between the instructor and the students.

Today ME faculty stopped testing basic mathematical skills because majority of faculty feels it is the task of the mathematics department. Some are so focused on engineering applications that they would routinely provide students formulae to use and skip over the relevant mathematical concepts. Ironically, some faculty members feel that in the age of computers such analytical skills are obsolete! However, ME faculty must admit that unless there is discussion and return to the basics, a fluid mechanics problem would be incorrectly solved on tests, or at the very least, misunderstood. Forgetting analytical skills results in making engineering and computational blunders. Our intention is to strengthen the logical basis of thinking (whenever possible, let symbolic computing catch and correct the human processing errors [20]) and we do not wish to replace analytical thoughts at any cost. We collected some areas of confusion and presented in a table below. This collection came from actual tests and quizzes already tested and reported in [4] - [6], [19]. 
Learning mathematics-based ME courses have the following areas of confusion:

1. Confusion due to different terminology, units (cf. Figure 3)

(e.g., mass flow rate is measured as mass over time, whereas, heat flow rate is measured as heat energy over time and area)

2. Recall correctly the order of operations in mathematical expressions (cf. Figure 3)

3. Lack of practice in logical thinking [4][5]

4. Perceptual connectivity of fractions, percentages and decimal numbers [4]

5. Inability to recall essential algebra, geometry and trigonometry from high school mathematics [2][5]

6. Deficiency in pattern recognition of planar figures and mathematical expressions.

7. Lack of connectivity of tangential and cylindrical coordinate systems to Cartesian.

8. Lack of understanding in engineering approximations and assumptions [5]

9. Geometrical meaning forgotten from previously learned mathematical operations in differential and integral calculus.

Our experience shows that instead of introducing abstract and generalized theories, these examples must be created to serve an engineering courses' needs. We began data collection on mathematics-based courses in 2008 [see reference 6] but in the meantime RIT changed from a quarter-based to a semester-based system. Therefore, the ESCC program had to be restructured. Recently gathered data will be disbursed among the faculty soon so that a proper focus may be created by a gaged process of reinforcement through the engineering courses. Such data would be considered by the curriculum advisory committees. This paper proposes one such gaged measure and reports the initial feedback.

\section{Learning bottlenecks}

After gathering over ten years of data in this manner we have always been seeking better solutions. But we are constantly challenged by program changes. The first course in fluid mechanics is a prerequisite of the Fluids II course. 25 years ago, we would not allow a student to take the first course in fluid mechanics before receiving a satisfactory grade in Dynamics, for which Statics is a prerequisite. But now Fluids Mechanics is often taken in conjunction with Dynamics assuming that the needs for the Reynolds Transport Theorem would be furnished with enough of rigid body dynamics coverage simultaneously. Often this causes a learning bottleneck because students may not have seen enough of rigid body dynamics and neither have felt quite comfortable with sketching both shear and pressure forces on an isolated fluid control volume (CV). While sketching a CV the streamline that has to be sketched separately on the centerline of the fluid volume by choosing two points on it, which often gets overlooked by students as a requirement to apply the Bernoulli equation. To complete the background necessary for Fluids II, a first course in heat transfer is a mandatory co-requisite (or, students who took it before would 
also qualify). Thermodynamics is a pre-requisite for Heat Transfer. Thus, the flow of courses from Statics and Thermodynamics to Fluids II seems feasible to build a solid foundation for our students. Unfortunately, if the CV methods are not learnt well in Fluid Mechanics, development of Fluids II differential formulations of fluid and thermal boundary layers suffers a major setback. Similarly, if students did not learn the algebraic similarity ideas offered by the Buckingham's $\pi$-theorem they may find it difficult to understand the flow analogies presented in Fluids II, which must provide the basis for the Fundamentals of Computational Fluid Dynamics (CFD). A complete review of all fluid flow equations by the CV methods together with necessary principles of statics and dynamics is conducted in the first two weeks of the Fluids II course. This solves the preparedness check for our dual degree students who choose CFD as their terminal elective. The traditional MS students take the sequence of Ideal Flows, Convective Phenomena and CFD in their thermal-fluids concentration before thesis work.

\section{Sample results}

Here we used a 10-step approach to reach the terminal CFD course in our program. Some samples were reported before [6], [19]. The newly organized small steps not only provide coherence but have built-in motivational incentives also. They are:

1. Search topics of mathematical significance in the CFD course.

2. Trace in which engineering science course it was first introduced.

3. Study mathematical/conceptual preliminaries necessary to understand the topics upon their first introduction from a student viewpoint. For example, operations of exponential, logarithmic, transcendental, hyperbolic, and inverse functions and their properties would be necessary for review to develop connectivity of topics. Refreshing connectivity with geometries help also. 4. Prepare a set of course notes with complete details of the mathematical preliminaries with worked out examples. Students are strongly urged to read these notes along with their textbook. 5. Discuss course topics that assist recall of related concepts, while offering explanations and details where the text may have skipped several steps to achieve the result.

7. Design examination and quiz questions emphasizing the concepts during reviews.

8. After grading, promptly return papers to students with comments stressing areas to overview. However, do not post solutions yet.

9. Allow students to brainstorm the missed concepts with the help of instructor provided notes and allow them to resubmit the examination or quiz after relearning the topics to obtain better grades. Find average of each new grade and the original grade and replace the original grade by the average.

10. Strictly grade each individual re-submission and expect each answer to be $90-100 \%$ correct mathematically and procedurally. Otherwise grades are unaltered. Then post the solutions with your summarized comments to students why some students were not given better grades.

The regrading offer [cf. Appendix] is a great motivator. Although the steps $8-10$ above take extra efforts by the instructor teaching the course for the first time, most students appreciate the efforts made by the instructor as pointed out before. Most importantly, the efforts consistently 
improved class averages of over $20 \%$ on quizzes and examinations for the past three years. The above approach is utilized for our undergraduate program only. However, by tracking students who take the graduate Convective Phenomena later we confirmed that they also retained the concepts better.

Multiple pathways to the same solution must be clearly explained. These points are elaborated further below (see Figure 5). In this question taken from reference [24] (modified by added mathematical notes) students are forced to find and sketch streamlines in ( $r-\theta)$ coordinates (for realizing the benefits of such a coordinate system over a Cartesian coordinate system). Allow students to try both systems. Ask them to apply superposition first using stream functions, and then using velocity components. Many students would not know how to add or take derivatives of inverse tangent functions. Review these. Also introduce concepts of hodographs as future study areas which would come a bit later as students mature. In this way, a single problem may review several mathematical concepts.

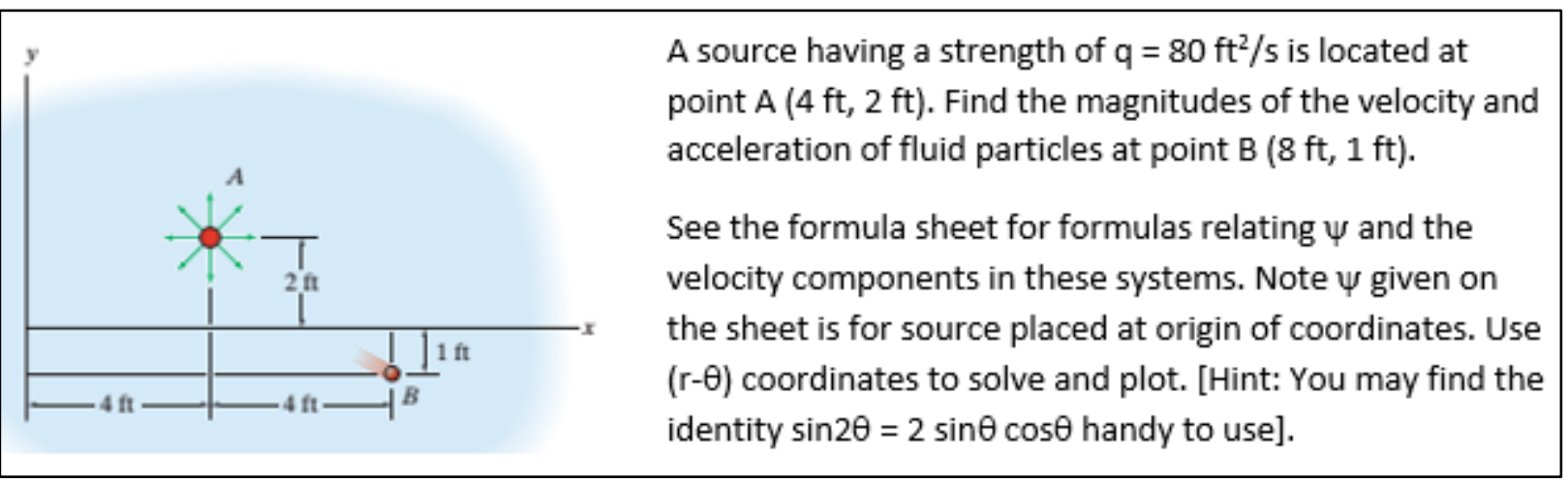

Figure 5. Mathematical ease of using a cylindrical system over a Cartesian system

Continue further with ideal flows and establish a logical thought pattern illustrating the differences between rotation in solids and fluids. We use the archived movie Vorticity [25] together with class examples for this purpose. The instructor asks students to learn from the movie (and the concepts therefrom) very much like what a flipped class would ask. However, this happens only after the movie is screened in class with complete explanation of the concepts by the instructor. For example, Fluids II syllabus does not cover Crocco's theorem and Kelvin's theorem. Crocco's theorem relaxes the stringency to use Bernoulli equation only on a streamline. Several types of conceptual idealizations practiced by engineers are also learnt from the movie. Discuss with contrasting examples how pressure distribution as obtained from application of Bernoulli (or Euler's equation) would yield the same solution as obtained from the superposition of ideal flows. Students must understand the concepts of contrasting experiments depicting the shear and rotation behaviors. Therefore, Laplace solution in $\psi$ or $\phi$ for low-speed flows which bypass solving the nonlinear Euler's equation may fully be understood with advantage in design concepts [9]. Ask students to quote such examples on quizzes and discussions. Extending the discussion to volume expansion and geometrically presenting the dilatation completes the picture without using the solenoidal jargon or tensors. 
We emphasize the Reynolds Transport theorem as the single synthesizer for understanding all fluid flow conservation laws (very much like Newton's laws are the synthesizers for both Statics and Dynamics). After developing the differential equations from infinitesimally small CVs, a preview is offered to students how these may be utilized for marker and cell techniques in CFD problems [6]. Finally, when matrices are introduced in CFD, students truly get the complete picture of what we mean by solvability of an engineering question from the beginning to the end including realization of assumptions and constraints. All 3 types of formulations (e.g., lumped, differential and integral [23]) are completely reinforced in the CFD course.

We recommend the following steps of mathematical rigor to overcome the learning pitfalls:

1. Introduce a complete discussion of the limit process of how to shrink a volume to a point or changing a secant to a curve to a tangent line demonstrating orientation of the $\overrightarrow{d r}$ vector (so that they may understand how $\vec{V} \times \overrightarrow{d r}=\overrightarrow{0}$ represents a streamline) complete with implications in $\mathrm{n}-\mathrm{t}$ and $\mathrm{r}-\theta$ systems.

2. Details of Taylor Series order of approximations with actual numerical examples when the order definition may not hold.

3. Overview of summation formulae for common transcendental functions, and establish the connectivity with compound angles (e.g., how $\sin (\mathrm{A}+\mathrm{B})$ may be used to yield $\sin 2 \mathrm{~A}$ or, $\sin 3 \mathrm{~A}$ ) 4. Clearly establish the understanding of a well-posed problem using a lumped, differential or integral approach.

4. Sketch a tiny volume and write conservation laws using first order approximations, then show the transition to differential equations including dividing by the volume before limits. As an example, each term of Navier-Stokes equation for incompressible flows expresses force per unit volume. Continue with simplifications to various applications in two and three dimensions.

5. Discuss concepts in creation of a boundary condition by using a stretched CV.

6. Thoroughly review dimensional analysis for algebraic and differential equations.

7. Discuss regimes of fluid flows beginning each time with the conservation laws.

Do not allow students to make any assumptions that are not necessary. The assumptions memorized are not understood. Some instructors state assumptions immediately after starting an engineering solution, which should be avoided. If instead students discuss in groups the advantages and disadvantages of making assumptions (help them understand the connectivity to formulations), assumptions would be remembered and recalled more easily. It is necessary to create a few counter examples for this purpose. For example, instead of assuming a fullydeveloped flow it is better to assume a parallel flow and use it consistently to simplify continuity and momentum equations. Give practical examples of dimensionality and connections to velocity components.

\section{Recommendations for ESCC faculty}

1. Search which course or courses may reinforce mathematical concepts. 
2. Open a faculty dialog in the program to see if other faculty members teaching elective courses may be willing to participate in the reinforcement process. Emphasize that without the reinforcement, the topics would be lost soon.

3. Monitor performance improvements and learning willingness in students (see appendix). 4. If necessary adjust course grades when students are willing to improve themselves. Re-grading requires a willful participation of students to relearn from the distributed resources.

The above process also prepares better future faculty members.

"Rarely, if ever, do we concern ourselves with the process of education, the long-term effectiveness of our efforts, the instrument of learning (i.e., the student), and the anatomic/physiologic constraints that impede or enhance both the learning and the teaching process..."

\section{Discussions}

In this research we have documented some experiences with different aspects of CBL. We blended experiential and team-based learning in our revised approach. Most literature related to engineering education practice in the United States overlooks an important fact. For example, reference [15] states "in this system of instruction (referring to CBL), students cannot advance or be evaluated for a new competency until they have mastered the prerequisite materials. Thus, students receive differentiated support based on their pace of learning". For some time, RIT adopted a similar strategy of mathematics instruction. But our experience shows that weak students need to understand mathematics more than simply use formulae to solve an engineering question. Does it really matter to the average students (who do not have enough time to master topics)? We (faculty) must assist students to achieve connectivity and implement self-learning practices at the same time. It is therefore important not to overwhelm them. One drawback of CBL is compartmentalization. Since learning never happens in a straight line, it is important to see the global picture, while understanding why the assumptions are relevant to simplify models. A formulation may never be fully understood by picking the correct equation from a set of alternates. However, such a form of multiple-choice question is often chosen by some ESCC faculty. Our experience shows that students often pick incorrect equations later from ME handbooks and the Internet. Instead we recommend that time would be well-spent to train students why and how to construct mathematical equations from some simple concepts. In this paper we described a scaffolding approach which stretches time and motivates students to become self-learners.

\section{Conclusion}

The axioms of learning are quite different at the undergraduate from the graduate level. For example, research-based exposure may be delivered successfully to highly motivated undergraduates with excellent mathematical background. For them, the APOS [16] theory would be ideal for describing stages of learning. It is fully researchable for developing instructional 
strategies. On the other hand, Perry's scheme [23] is not fully describable from an analytical viewpoint. But a "nudge is always the best". From the student perspective, instructional delivery expectations and measure of student efforts have become major contributing factors on the retention and demonstration of learning $[17,18]$. Therefore, the process adopted in this paper is a careful blend of pedagogy with mathematical interpretations of fluid mechanics problems and connectivity achieved through a time-tested, motivational reinforcement process. We hope that students trained using our approach would be less afraid to face any analytical challenges.

\section{Acknowledgments}

This study would not have been possible without the active support of all members of the ESCC instructional teams for the past nine years. All participating faculty members and trained teaching assistants helped in designing, discussing, and evaluating procedures discussed here. The author, who was also the ESCC coordinator in the past, gratefully acknowledges all their contributions. The author would also like to thank Drs. E. C. Hensel and R. Robinson for helpful discussions.

\section{Appendix}

Each quiz or test given in the Fluids II class is designed to test individual proficiency. However, over the years while experimenting with group learning [4], [6] we discovered a strong grading incentive that works well with students. Therefore, our proposed course structure used an effective combination of group learning and specially prepared detailed course notes. After the first (background check) quiz the following e-mail (boxed below) was sent to the class giving students another opportunity to relearn the topics. The quiz 1 mentioned below was multiplechoice type. Many students would guess answers on such questions. But the condition for regrading such quizzes was they must learn the correct reason/s for each of the missed question by reading notes, or by discussions with groupmates or others. This worked very well. In fact, our data shows a consistent interest among students. Further tracking some of the students from Fluids II to the Convective Phenomena course, we found they retained these relearned concepts later, and thus have performed well on the background check quizzes. The e-mail read 
As I mentioned in class today, we begin the course with a quick recall of topics which must stay in your active memory when you learn Fluids II. The first quiz that you took in class today is a representative sample of the topics that would be necessary for you to recall. Each of these topics is available (if you search the $550 \mathrm{CD}$ over the weekend) from myCourses. This is why I have placed a blank quiz in a folder called "Quiz Blanks" on myCourses. You may have missed to answer some of the questions correctly in today's quiz. But if you relearn these topics over the weekend, print out a copy of the quiz and return your corrected quiz with reasons why you answered now differently.

For example, question 1 is related to the definition of a Newtonian fluid. In this semester all flow questions attempted would involve incompressible flows. So you must know what type of flow it involves. Similarly, we'd solve differential equations with assumed velocity profiles. So you must know what that involves in question 4, etc. If you demonstrate to me that you have learned the topics correctly now, I promise to average your newly earned grade with the original grade received and replace your original score earned on my grade book. Note that grade correction is only possible if your new score is more than your original score. So find out the correct reasons for the answers by the time you re-submit.

Some samples of student comments from ESCC Course Evaluations after Fluids II:

I think his grading appropriately reflects his teaching/testing style, which I like. Dr. Ghosh wants people to understand his material on an intimate, conceptual level which requires tests and quizzes be more difficult because you cannot simply rely on knowing formulas. He therefore is quite lenient with grading and awarding points to wrong answers that he believes were conceptually on the right track. He asks a lot from his students but does not punish a more difficult style of teaching/learning with harsh grading, if that makes sense...

I love the fact that we can do the quiz and test corrections. That's great, because it encourages us to go back and understand what we got wrong, and the reason we missed a question. I think that is a much better way to grade and motivate/encourage learning, than not offering corrections. The lectures are very interesting. I finally started to feel pretty comfortable with a lot of the calculus and math concepts behind a lot of engineering in this class because Dr. Ghosh explains it so well (and spends so much time on it). The 550CD notes are a really powerful reference. I loved the movie that he showed in the beginning of the semester. It really helped, have a physical understanding of fluid mechanics and understand the conventions and math principles. I really appreciate how Dr. Ghosh focuses on making sure you understand the physical reasons behind the math and formulas.... 


\section{References}

[1] R. C. Hibbeler, Engineering Mechanics - Dynamics, 14 ${ }^{\text {th }}$ Edition, Pearson, 2016.

[2] A. Ghosh, "Foundations of Statics - An Assessment Study and Feedback Implementation", Paper No. IMECE 2016-66302, ASME International Mechanical Engineering Congress and Exposition, November 11 - 17, Phoenix, Arizona, 2016.

[3] A. Ghosh and E.C. Hensel, "An interpretive assessment of engineering science core courses", Paper No. IMECE 2009-12939, ASME International Mechanical Engineering Congress and Exposition, November 13 - 19, Lake Buena Vista, Florida, 2009.

[4] A. Ghosh, "Teaching Formulation Skills in an Upper Level Fluid Mechanics Course", Paper No. IMECE 2011-63989, ASME International Mechanical Engineering Congress and Exposition, November 11 - 17, Denver, Colorado, 2011.

[5] A. Ghosh, "Formative Assessment using Multiple Choice Questions in Statics and Dynamics", Paper No. IMECE 2016-66304, ASME International Mechanical Engineering Congress and Exposition, November 11 - 17, Phoenix, Arizona, 2016.

[6] A. Ghosh, "Development of Analytical Skills through Cooperative Learning", Paper No. IMECE2009-12947, ASME International Mechanical Engineering Congress and Exposition, November 13 - 19, Lake Buena Vista, Florida, 2009.

[7] R. DeLoach, "The Modern Design of Experiments for Configuration Aerodynamics: A Case Study", 44th AIAA Aerospace Sciences Meeting and Exhibit, Reno, NV, USA, 2006.

[8] D. L. Ashby, "Potential Flow Theory and Operation Guide for the Panel Code PMARC_14", NASA/TM-209582, 1999.

[9] J. McCune, H. Wachman, and E. Murman, "A Workshop on Teaching Fluid Mechanics with Workstation Based Software”, Final Report, National Science Foundation, 1990.

[10] F. Stern, T. Xing, D. Yarbrough, A. Rothmayer, G. Rajagopalan, S. P. Otta, D. Caughey, R. Bhaskaran, S. Smith, B. Hutchins, and S. Moeykens, "Hands-On CFD Educational Interface for Engineering Courses and Laboratories", Journal of Engineering Education, 95(1), January 2006.

[11] J. Cabral, "Is Generation Y Addicted to Social Media?", The Elon Journal of Undergraduate Research in Communications, 2(1), 5-14, 2011.

[12] D. Perkins, "Theories of difficulty" in Student learning and university teaching, eds. N. Entwistle and P. Tomlinson. British Journal of Educational Psychology. Monograph Series II: Psychological Aspects of Education - Current Trends, 2007.

[13] Carr, D.H. Thomas, T. S. Venkataraman, A. L. Smith, M. A. Gealt, and M. T. R. Quinn, "Mathematical and Scientific Foundations for an Integrative Engineering Curriculum", Journal of Engineering Education, Volume 84, Issue 2, April 1995. 
[14] T. A. Litzinger, J. C. Wise, and S. H. Lee, "Self-directed Learning Readiness Among Engineering Undergraduate Students", Journal of Engineering Education, Volume 94, Issue 2, April 2005.

[15] M. Henri, M. D. Johnson, and B. Nepal, "A Review of Competency-Based Learning: Tools, Assessments, and Recommendations”, Journal of Engineering Education, Volume 106, Issue 4, October 2017.

[16] I. Arnon, J. Cottrill, E. Dubinsky, A. Oktac, S. R. Fuentes, M.Trigueros and K. Weller, APOS Theory - A Framework for Research and Curriculum Development in Mathematics Education, Springer, 2014.

[17] F. E. Ritter, G. Baxter, J. W. Kim, and S. Srinivasmurthy, "Learning and Retention", The Oxford Handbook of Cognitive Engineering Edited by John D. Lee and Alex Kirlik, February 2013.

[18] J. W. Kim, F. E. Ritter and R. J. Kobek, "An integrated theory for improved skill acquisition and retention in the three stages of learning", Theoretical Issues in Ergonomic Science, Volume 14, Issue 1, 2013. https://doi.org/10.1080/1464536X.2011.573008.

[19] A. Ghosh, and C. Pantaleon, "Teaching Computational Fluid Dynamics Using MATLAB" Paper IMECE-66458, International Mechanical Engineering Congress \& Exposition, San Diego, California, USA, Nov. 15-21, 2013.

[20] C. Pantaleon, and A. Ghosh, "Taylor series expansion using matrices: an implementation in MATLAB”, Computers \& Fluids, Volume 112, 2 May 2015, Pages 79-82, 2015.

[21] M. G. Schrlau, R. J. Stevens, and S. Schley, "Flipping Core Courses in the Undergraduate Mechanical Engineering Curriculum: Heat Transfer”, Advances in Engineering Education, volume 5, no. 3, Fall 2016.

[22] L. Abeysekera and P. Dawson, "Motivation and cognitive load in the flipped classroom: definition, rationale and a call for research", Higher Education Research \& Development, Vol. 34, No. 1, 1-14, 2015, http://dx.doi.org/10.1080/07294360.2014.934336

[23] P. G. Love and V. L. Guthrie, "Perry's Intellectual Scheme”, Chapter from Understanding and Applying Cognitive Development Theory: New Directions for Student Services, No. 88, Jossey-Bass Publishers, San Francisco, 1999.

[24] R. C. Hibbeler, Fluid Mechanics, $1^{\text {st }}$ Edition, Pearson, 2015.

[25] A. H. Shapiro, "Vorticity - Parts I \& II", National Committee for Fluid Mechanics Films, MIT Press, 2008.

[26] V. S. Arpaci, Conduction Heat Transfer, Addison Wesley, 1966.

[27] D. J. Schneck, “Integrated Learning: Paradigm for a Unified Approach”, Journal of Engineering Education, Volume 90, Issue 2, 2001. 